\title{
Review of: "SARS-CoV-2 is localized in cardiomyocytes: a postmortem biopsy case"
}

\author{
Ahmet $\mathrm{Oz}$
}

Potential competing interests: The author(s) declared that no potential competing interests exist.

\section{Reviewer 1}

Dear author;

I reviewed the case report entitled 'SARS-CoV-2 is localized in cardiomyocytes: a postmortem biopsy case'. I found the case very interesting and useful for our journal. However, I have some minor comments before the acceptance of article.

--minor comments;

1-There are some grammatical mistakes in the article, hence, I recommend the proof-reading

2- The discussion section in the article is a lit bit short; therefore, it should be increased. 